\section{MINUS3 - A GENERAL PURPOSE DATA ACQUISITION SYSTEM AT LBL'S 88"-CYCLOTRON AND SUPERHILAC*}

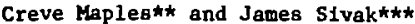

\section{Abstract}

MINUS3 is a general, multi-taked data acquisit1on package operating on the ModComp IV/25 computers at both the $88^{\prime \prime}$-Cyclotron and SuperHILAC. It currently can acquire data via three different channels: Interrupt; serial DMA 1Ink; and remote slave units for hlatogram type data. Two additional acquisition paths, CAMAC (with programmable differential branch drivers) and MODACS (for multiple CPU linkages and control) are scheduled to be alded in the near future. The package operates In a prioritized, time-available mode which permits It to dynamically adapt to microscoplc data rate structures due to beam characteristlcs at different acceleralors. Spectal hardware has been added to the graphics system to provide enhanced high-speed interactive capability. The program framework is also designed as a parasitic environment in which users may, in parallel, attach their own specialized and independent code.

\section{Introduction}

The software package MINUS3 1s currently ut1lized for the acquisition, control, and on-line analysis of data from experiments performed with both the $88^{\prime \prime}$-cyclotron and SuperHILAC accelerators at the Lawrence Berkeley Laboratory. The $88^{\prime \prime}$ Cyclotron is a mult1-partfcle, variable-energy, sector-focused cyclotron. At present it is capable of accelerating protons to $60 \mathrm{MeV}, 11 \mathrm{ght}$ tons (. 4 4) to about $35 \mathrm{MeV} / \mathrm{A}$, and heavy lons to er ergles of $140 q^{2} / A$. From the point of view of daza acquisition, the cyclotron produces an essentially continuous beam. Thus, for a given experiment, the data rates are roughly constant and continuous.

The SuperHILAC is a heavy ion Inear accelerator capable of accelerating lons (up to ${ }^{29 \theta} \mathrm{Pb}$ ) to energles up to $8.5 \mathrm{keV}$ per nucleon. It is a pulsed machine giving 36 pulses per second, each approximately 4 msec in duration. The accelerator also permits the time-sharing of beams of different lons into different experimental areas. Data acquisition at this facilicy therefore requires handling intense bursts of information approximately every 30 msec.

The types of experiments carried out at both accelerators vary greatly and include polarization work, gamm-ray studles, nuclear particle opectroscopy, and investigation of nuclear reaction mechanisms. Historically most research groups independently handled their own acquisition problems, often whth their own computers, In 1975 ,

*Supported by the Nuclear Physics Division of the U.S. Department of Energy.

**Lawrence Berkeley Laboratory, Un1vergity of California, Berkeley, CA 94720

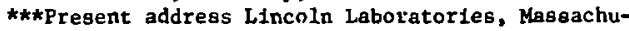
setts Institute of Technology, Lexington, Mass. however, a decision was made to attempt to standard1ze the problem of data acquisition and handling at both accelerators. This standardization includes using the same computer, acquisition hardware, and software framework for the majority of experiments.

\section{Computer Configuration}

One of the first steps in standardizing the acquibition environment was the decision that, during on-1ine data acquisition, the total resources of one computer would be devoted completely to the experiment. This computer would then be expected to handle data acquisition and data storage as well as to provide all the tateractive control and real-time processing (including monitorIng, analysis, and display) required. The decision to utilize one dedicated computer for acquisition was based on the belief that the on-line demands on the CPU, particularly in the area of real-time, event-by-event analysis, would be substantial and would continue to increase. Indeed, it was felt future requirements, both in acquisition and in on-line analysis, might exceed the capability of a single computer.

The selection of ModComp IV/25 computers was based primarily on their multi-task hardware features (e.g., hardware mapping and resister saving), the avallable map space ( $131 \mathrm{~K}$ bytes/task), the memory capacity ( $524 \mathrm{~K}$ bytes), and the ability of the CPU to perform fast 32-bit integer operations. Three of these computers came on-line in the sumer of 1976. Currently four of these computers are in use and a fifth, a new ModComp classic, is being installed. In order to maintain complete compatibility berween the computers and to run standard operating systems and acquisition software, all systems are configured with the followfing minfmum hardware:

1. $328 \mathrm{~K}$ bytes of memory

2. Three, 2.5 Mbyte cartridge discs

3. Two magnetic tape drives (75ips)

4. Hardcopy operator console

5. Specially modifled Tektronix 4014 graphics terminal

6. Versatec $1000 \mathrm{LPM}$ printer/plotter/copler

7. Standard data acqutsition hardware (discussed in the following section).

The Tektronfx 4014 terminals have been speclally modifled to provide better interactive and display features. The keyboard portion of the terwinal has been lengthened 2 inches by inserting a machined plate containing 15 physical switches at the base of the storage screen (figure 1). These switches, some of which are two position, together with 4 standard switches on the Tektronix 4014, have been interfaced to the computer to provide 31 readable owitch positions. The switches themselves have no intrinsic hardware function but are monttored by software to provide a wide range of functions depending on the prograt executing. In the acquisition environment the switches provide features 


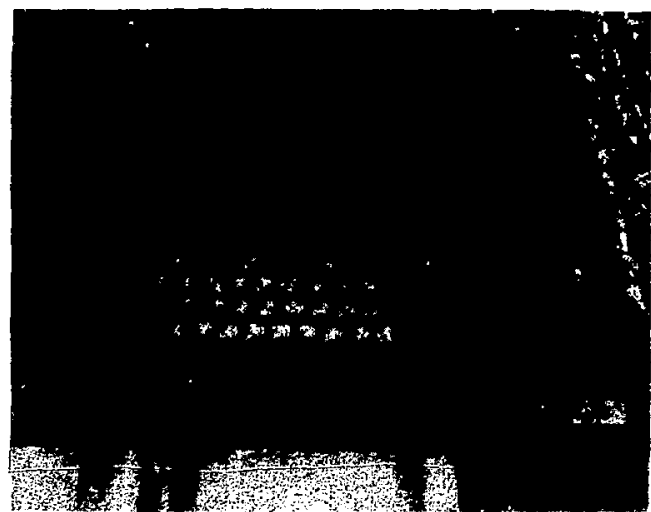

Fig. 1. Tektronix 4014 keyboard modifled to include a set of interactive switches. (XBB772-1179)

ranging from simple $x, y$, or $z$ scale manipulation to dynamic interactive background fitting and multiple peak deconvolution. In addition, eacin switch also contains an independent light which is also operated under software control. These lights may be used for any function, such as inting to ladicate user action is required, but in a typical acquisition enviranment they are employed as a user ald. For any given combination of switch settings, the lights will Indisate what remaining user options are avallable.

An additional "safety" switch was also added to the terminal. The function of this switch is to disable some standard hardware features of the 4014. In particular, these include the line/local switch, the teletype-lock key and the margincontrol switch. This modification protects the user against accidentally altering any of these switches which would normally change or suspend the operation of the scope. The installation of this safety device resulted in about a $75 \%$ reduction in the number of reported hardware problems.

The standard transmis..ion rate of graphics information from the computer to the terminal ( 9600 baud) also necessitated some hardwere additions. Th1s transmission rate proved far too slow for effective interaction with the scope. This Is especially true when high density plots (multiple 1- and 2-dimensional displays) are involved. (See sections on HYDRA and SPACE.) To alleviate this situation a special direct memory interface connection was constructed between the main computer and the 4014 terminal. with this link, graphics information can be transmitted at rates equivalent to approximately $120 \mathrm{~K}$ baud.

\section{Data Acquisition Hardware}

For purposes of description, the data acquisition hardware will be divided into three classes:

1. High-Speed Event Recorded Data

2. Histogram Data

3. Periodic or Low Speed Information.
Event-by-event data have traditionally been acquired by using a multiplexed ADC system to frovide up to 16 channels of information per event. The ADC provides 12-bit accuracy with a fixed conversion time of $30 \mathrm{usec}^{2}$ The multiplexer ${ }^{2}$ provides the ability to stretch signals up to 1 msec with stability of better than 1 part in 5000 . Data acquisition ucilizing this setup is carried out over a high-speed serial link to the computer. Using this acquisition channe], data are acquired in an interrupt mode, with the CPU processing one Interrupt per parameter. The maximum data collection rate, epproximately $18 \mathrm{~K}$ parameters/sec, is 11mited primarily by the speed of the front end elcctronics.

in order to achleve higher data rates and to decrease the CPU overhead in data processing, a DMA data acquisition interface has been cotstructed, integrated into the system, and is in the final it age of testing. This channel was designed so that it could operate with new fast 12-bit $A D C$ units, having fixed conversion times of about 5 usec. With this channel the maximum acquisition rate is expected to be between 50 and $70 K$ parameters/sec, but inpractice will be limited to less than this by tape storage speeds.

It is anticipated that in the near future it will be necessary to process more than 16 parameters/event. At this point it will become advantageous to utflize data acquisition hardware different from the tràditional multiplexed $A D C$ approach. Although the DMA channel is not limited in the number of parameters/event it can handle, it appears that it may be economically more feaslble to move to a CAMAC based environment. The Initfal cost of such a conversion may be substantial considering nelther accelerator currently operates with CAMAC. It is hoped, however, that In the long run, and with a large number of parameters/event, this solution will be more cost effective and flexible. To this end, it was declded to utilize the Differential Branch Driver (DBD) interface developed by the Los Alamos Sclentific Laboratory. ${ }^{3}$ This unit provides up to 8 DMA channels to the computer, each controlled by a separate instruction stack in the DBD. One such untt has been purchased and Installed on one of the ModComp IV/25's and is currently being tested and integrated into the system. The inclusion of these three modes of event-by-event data acquisition in the operating environment is schematically shown in figure 2. The software impact will be discussed in the section on CHAOS (no pun intended).

The necessicy of being able to process highspeed histogram or singles type of Information was recognized in the initlal system design. This type of data results in adding one to some memory location in order to create various individual spectra, as opposed to independently recording every event as described previous...y. Since this type of data can run at very high rites and can be required in addition to event recording, it appeared simpler and more efficlent to move it fron the main CPU to an external processor. Fitgure 2 schemgtically indicates the tho methods, analyzers or external memories, by which such information t. handled. Both devices of $\mathrm{fer}$ the common 


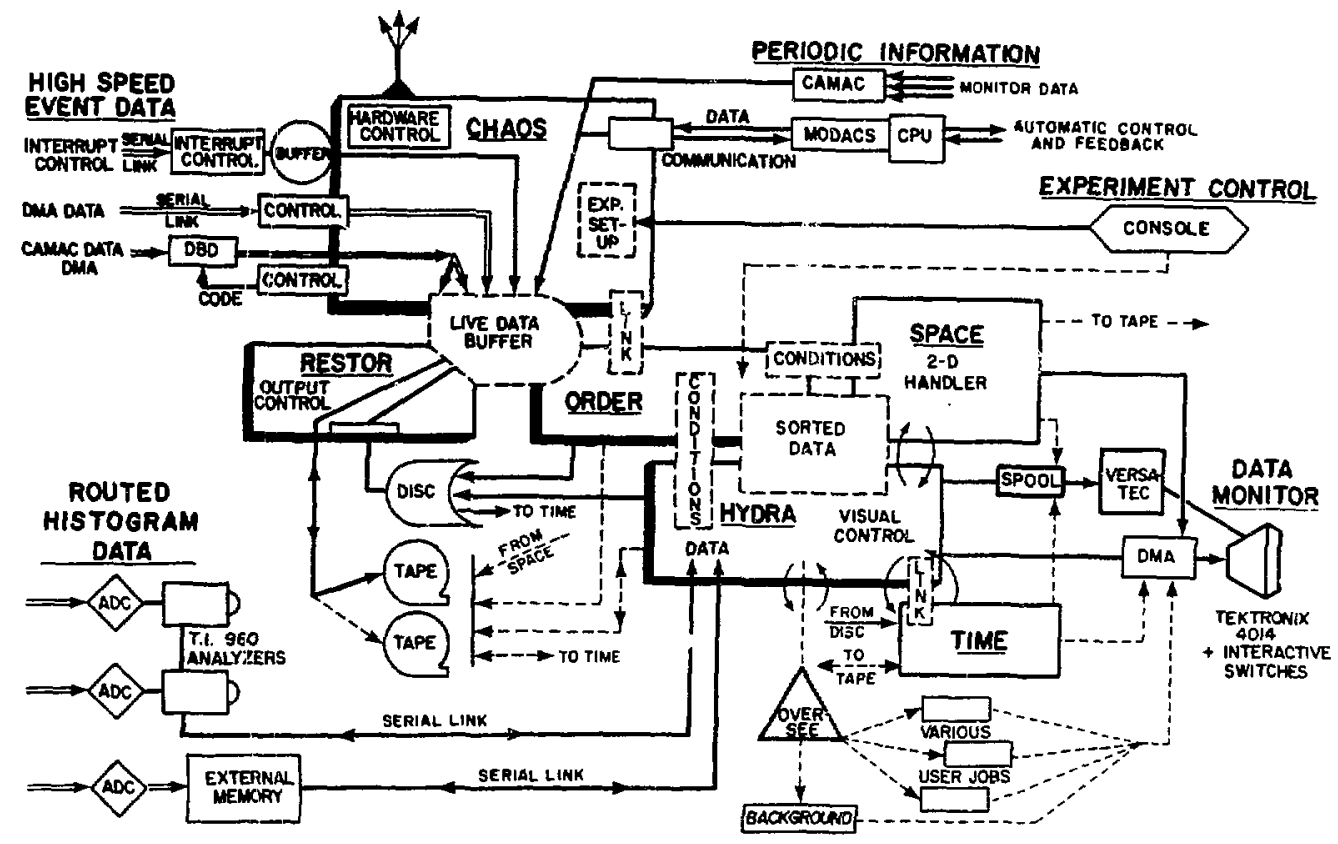

XEL Tou.1011

Fig. 2. Schematic layout of the MINUS3 data acquisition software wich associated hardware.

Ce rability of accepting routed ADC laputs to create mu. t1ple spectra of the desired resolution (12-bits maximum) within their oun local memorles. In both cases 24-bit memory is used and the units can operate in the add-one mode at speeds of about 300 nsec per data word. The external memory is simply an extemal block of buik memory with a specialized interface. The analyzers are bullt around the TI960 processor and provide many local and traditional analyser functions, such as a small display rireen, peak integration capabllity, etc. More rhas on analyzer may be coanected at a time. In this manner a user can have both a local display at $t$ ti analyzer as well as a more versatile dynamis displiy on the computer graphics terminal. Both analyzers and external memorles are connected to the computer through a serial link and may be controlled and dynawically monitored by the main computer.

A final class of data which must be handled is the acquisition and control of periodic information. This could include such items as scalers, pressure or temperature monitoring devices, scatter chambe: positions, eagnet currenta, etc. This class of Information is characterized by being sampled at much lower rates, typically at some predetermined time, count, or interrupt determined interval. In addition some positive action by the computer, such as clearing scalerk, may also be required. Figure 2 Indicates two proposed channels to handle this type of information. One is simply a low speed
CAMAC 11nk, probably identical to the previously mentioned high-speed event link, but utilized oomewhat different1y. The MODACS link is a ModComp supplied unit designed both for dynamis control and to connect multiple cPU's. This link will therefore be utilized in situations demanding more involved type of control and feedback of dedicated equipment, Including closed loop control, The CPU will be set up initlally by the main computer and will subsequently supply status and monitor information as requested. Further discussion of the use of these units is contained in the section on TIME.

\section{MiNUS3 Acquisition Päckage}

The general design goal was to provide the following capabilities:

1. Versatility--The package must be able to accomodate a wide range of experimental requirements.

2. Direct interaction--The package must permit users to inceract directly with the realtime graphics display of data to obtain information or transform spectra.

3. Dynamic control--The user should be able to eusily and rapidiy alter sorting condtions on the live data and to examine new projections at will. 
4. Flextbility--The user should be able to easily and rapidly alter monitor condirions. This includes, for example, the creation of new monitor conditions or the utilization of different transformation finctions on the data to create new artificial parameters.

5. Ease of tusage--The package should be very easy to use. This is particulariy inportant alace considerable use of the packege is anticipated by outbide user groups who are only precent at LBL for relatively brief perlods.

6. Simpte user interfaces--This would permit specialized user software to be easily ats.ched to the existing framework to provide a wide range of highly specialized services.

7. Safety--This requires that all Interaction with the package be carefully examined to minimize the possibllity that input errors could lead to program crashes. Further, effort should be made to localize functions such that errors occurring during acquisition affect only local tasks, which way be restarted, and not the acquisition stream. Finally, all critical areas should be monitored and any malfunction clearly reported to the user and approprlate action taken (e.8.. stopping the experiment and sounding an alarm).

With these goals in mind the multi-task, software acquisition package called MINUS 3 has evolved over the past three years. The structure of the package is shown schematically in flgure 2. The finction and interaction of each task will be described in the following sections.

\section{CHAOS}

The function of the task CHAOS is to handle all proalemo assoclated with direct event acquisition and the control of related hardware. The tank interacts with the user via a hard-copy terminal and permits the selection of the type of data acquisition hardware to be used and, in the case of CAMAC, the configuration of the equipment. During acquisition one of $t:$ : functions of the code is the allocation of bufisi space for incoming data. In order to effectively handle a wide range of data rates, the size of each acquisition buffer is not deternined by the size of the tape records being written, but rather is dynamically adjusted accordIng to the actual data rate. The purpose of this ieature is to permit examination or analysis of the data as quickly as possible. In the case of low data rates, fon example, the individual input buffers may be only 200 words $100 \mathrm{~g}$. Each buffer is then rapidly avallable for analysis and dieplay. Even a rates of only a few event per second, users may quickly view the incoming deta rather than wating for a long 1000 word tape record to be filled. At higher rates the buffer ire is increased to a maximum of one tape record to optimize the transfer and minimize CPU overhead.

At present it is planned that chios will also handle the acquisition and Inclusion of periodic Information, euch as scalers, pressures, currents, etc. Th1s information is typlcally read on deand, at fixed-time or -count intervals, or by triggering an external low-rate interrupt. These monitoring conditions, if desired, would be requested and described by the user initially. CHAOS would then -elect and reserve appropriate space in the outgoing data buffer to store this information as it is obtained. As each plece of periodic information is processed a label will be associated with it indicating the time at which it was acquired. Hore complex monitoring and control functions will be handled by amall dedicated CPU via * ModComp MODACS link as indicated in figure 2. In this case code and/or user defined monitor conditions will be down loaded into the euxiliary CPU. This unit will directly carry all necesary monitoring functions, including feed-back control if desired, and will supply the information to CHAOS as requested.

Once data acquisition is underway CHAOS must, If necessary, handle the synchronization of the atorage and sorting tasks, RESTOR and ORDER. This synchronization is discussed respectively in the sections describing these tasks. The major effect on CHAOS is the sttuation where acquisition rates exceed storage rates, resulting in lost data. Two methods are provided in this situation. In the default mode, if no buffers are avallable for incoming data, CHAOS will switch acquisition to a small secondary buffer. Data placed in this buffer will be lost. When the buffer is full a record will be kept of the number of events processed and a check made to see if output buffers are now avallable. If they are not, the above process is repeated. When acquistion is terminated a report is made to the user indicating the total number of events processed and the total number lost. Alternatively ChAOS can be instructed to terminate acquisition until such time as a buffer becomes avallable. In this mode the effective dead time would be determined by monitoring the external hardware.

The user interaction with CHAOS is conversational, normally question and answer. If a question is not understood or more information desired, a "?" can be entered. Aithough the primary function of the task is data acquisition, it also permits the replay of event data tapes. During acquisition or replay this task will not accept any direct input from users, and monitors only a few words of critical information from other tasks. Furthermore, during these operations there exists no path by which users may affect any Information utllized by this task. Th1s isolation is designed to minimize the possibility of fallure during eritical operations. ChAOS resumes user comunication in the following situations: termination of acquisition (this is accomplished by hardware commands issued by other tasks); by reaching the end of a data set being replayed; or by some malfunction (e.g., tape failure) detected by the KINUS 3 package.

Having determined the acquisition requirements of the user, CHAOS will activate the additional tasks required to handle data storage, analysis, monteor and display capability, and user interaction. 


\section{RESTOR}

This relatively simple task has the highest priority of any task in the package. Its function is to handle the starage and retrleval of eventrecorded information. The task, whith is normally suspended, is activated by CHAOS whe: a full tape buffer of data exists. RESTOR attempts to store this information according to user defined conditions. By default, the data are stcred on magnetic tape in 1000 word records, although different conditions can be specifled. The tape w:iting is carried out in a quick-return mode and all operations are monitored directly by this task.

The most 1mportant duty of this task is error handling. Before data acquisition may begin this i task must: 1) verify the condition of the storage unit, making sure it is both available and operational; 2) take exclusive use of the unit to prevent any other task from accessing it; and 3) verIfy that the unit is properly positioned for writing. This last requirement insurus that previously existing information is not accidentally destroyed. Before the recording of data is permitted, RESTOR checks that the unit is positloned at the logical end-of-medium. If this is not the case the user is Informed that the unit is mispiositioned and some action is required. Typical cholces would be to efther reposition to the logical end-of-medium ( $a$ single comband) or to intelalize the medium for rE-Use. Only after successfully writing a descriptive header record is acquisition peroltted to begin.

During acquisitiol RESTOR constantly monitors the writing process. Any detected transmission errors are handled by the task directly, not by the operating system. The task. will attempt to recover from simp.e errors (e.5;, parity) in both the acquisition and replay modes. Direct user communication with this task does not occur unless some non-recoverable problem $1: 3$ detected. In this event the task has the ability to 1modiately terminate data acquisition and to sound alarms. In all cases, however, the user is notified of the nature of the difficulty and is given the cholce of either terwinating the process or retrying the I/ 0 operation after attempting; to correct the difficulty. If the data buffer contains periodic information, RESTOR will copy that portion of the data into its own temporary biffer for subsequent storage on a special disc fil». After successfully processing a data buffer, the task indicates that this portion of memory is now avallable for reuse.

\section{ORDER}

The purpose of this task is to analyze or process the raw data in a manne defined by the user. The types of analysis capabilities include the following:

a) Definition of Artifical or Pseudo Parneters-This permits artifical parameter to be created which are functional transformations of the current parameter set. This feature will be discussed more fully below.

b) Creation of 1-Dimensional Spectra--Each opectrum may contain any number of channels up to a maximum of 4096. The channels for a given spectrum may be defined as consisting of either 2 or 4 bytes.

c) Projection of Gated 1-D Spectra--This permits the allacation of memory to be used for projecting information on a parameter accordIng to a set of conditions to be specified later. The actual specification of a given set of correlation conditions is normally an Interactive and dynamic function. (See HYDRA.)

d) Creation of 2-Dimensional Spectra--These are effectively plots of parameter $x$ vs $y$ with the $\mathbf{z}$ direction representing intensity. Any number of channels (up to 4096) may be selected for both the $x$ and $y$ axes. The only restriction is that the cotal amount of avallable memory cannot be exceeded.

e) Profection of Gated 2-D Spectra--This is similar to Item (c), bue the correlation conditions are used to project a 2-D spectrum.

f) Free-form or Functional Gating--Memory is allocated to peroit the storage of a 2dimensional free-form gate. The actual gate may be defined interactively at any time (see SPACE) and used as a projection condition (Item (c) or (e) above).

g) Scatter Plots--This provides the capability of plotting 2-parameter correlations on the screen directly and in high resolution (up to $4096 \times 4096)$. In this situation information is not stored in memory and exists only on the screen. Such spectra may, however, be used to set gating conditions in high resolution.

h) Biased Spectra--The program supports the capability of dynamically biasing both 1 - and 2-dimensional spectra. This enables regions of parameter space to be shown in greater resolution without the need for additional memory allocation.

1) Filtering-Filters are defined as sets of correlation conditions similar to those mentioned in part (c). However, they have the effect of removing information from the analysis stream (but not the data stream). This has the practical result of speeding up analysis.

The above list is intended to indicate the types of analysis currently supported by the task, or under development (items $f, 8, h$ and 1 ). The philosophy of ORDER is to make a block of memory (currently about 60K bytes) avallable for use in storing analysis results. The specific details of the utilization of this memory should, however, be completely determined by each user.

In addition to the actual analysis of data, it is important chat the task allow the user to add, delete, or change analysis conditions with a minimum of effort. ORDER will normally communicate With the user only on request (actually by a command to CHAOS). It is highly conversational and 
Is capable of processing either single or multiple commands. It provides guidence in its own operation by providing prompting information, by suggesting the next logical step, or by indicating what opt Ions are avallable. All input values are checked for consistency and the user informed of any discrepancy. In this way virtually all serous input errors are avolded. Modifications can be made during analysts setup in any order. Adding a 2-D spectrum to a given configuration, for example, requires only a 2 character command ("TD") and the 5 integer values requested by the task.

To achleve easy interaction it is normally desirable to minimize typed input. ORDER attempts to accomplish this in two ways: through the effective use of default values and directorled librarles of analys $\mathrm{s}$ conditions. With respect to defaults, MIN 53 will acquire data and produce spectra even if no analysis conditions are specifically given. In the case of changes or additions, ORDER will always assume the last used value of a paraneter if none is specified. In the previous example, the addition of a 2-D spectrum of the same size as the previous 2-D spectrun would only require typing 3 integer values. The task also permits analysis conditions to be stored or retrieved from labeled disc libraries on command. In addition, for safety, the current set of conditions is javed automatically on the completion of a setup and may be restored if necessary. Finally, the specification of analysis conditions is further similfied, where practical, by making conditions vartable and allowing them to be specifled as re ers siry in an interactive graphics envtronment. This inciudes such information as the number and type of gating conditions, the deterwination of their limits (Including free-form gates) and dynamically selecting spectrum blasing conditions.

The use of artificial parameters is a particularly important rool in analysis. ORDER offers three ways in which a user may achleve transformatlons of the basic data. A set of standard equations is directly available for transformations. It includes the following:

$$
\begin{aligned}
& \text { a) } P=(x / A+y / B+z) \\
& \text { b) } P=(x / A+y) \star \star B-(y) \star \star B \\
& \text { c) } P=(x+A) *(y+B) \star 2 \\
& \text { d) } P=1 /(x-A) \star \star 2 \\
& \text { e) } P=x /\left(A^{\star} x+B^{\star} y\right) \\
& \text { f) } P=A^{\star} x+B{ }^{\star} y+C \star(x+y) \star \star D \\
& \text { g) } P=A+B \star x+C x^{2}+D^{\star} x^{3}+E^{\star} x^{4}+F^{\star} x^{5} \\
& \text { h) } P=A * \operatorname{SQRT}(x) \\
& \text { i) } P=A * \operatorname{LOG}\left(B^{\star} x\right) \\
& \text { j) } P=A * \operatorname{Exp}\left(B^{\star} x\right)
\end{aligned}
$$

whers the varlables $x, y$ and $z$ refer to extsting parameters, $A, B, C$, etc., are constants and $P$ is the new parameter being created. For the purposes of speed, these calculations are elther carried out using inteper artthmetic or table lookup techniques. If tables are required, the necessary memory is obtained from the user's current display buffer.
If this basic list does not provide the desired traneforation, the list may be chained to produce more complex results. A new parameter, say number 12. for example, could be created using equation (a). This value could then be used as an input to Bnother equation, say as $x$ of equation (c). Further, If the intermediate calculation value stored in parameter $12 \mathrm{ls}$, by itself, not interesting, the final result of the second transformation could also be $s$ tored as parameter 12 . If the necessary transformation still cannot be obtained, the capability of directly attaching user written subroutines is currently being developed. In this case a user would maintain an independent library of routines which could optionally be attached when the MINUS 3 package was loaded. Basically these routines would be passed one event at a time and would return whatever transformations were desired.

It is clearly possible to have real-time data rates such that the requested event analysis cannot be carried out in the avallable time frame for all events. In order not to slow dow. the acquisition st ream the situation is handled in the following manner. ORDER maintalns a pointer into the live data buffer. One event is copled from this buffer beginning at the pointer location into a small second buffer and the value of the pointer updated. The specified analysis is then carried out on the secondary buffer. If, while this processing is belng carried out, CHAOS determines that this area of the live data buffer is needed for new data, ORDER's pointer is simply moved to a currently non-vnlatile area. After completing its current analys1s, ORDER will therefore obt in its next event at the new location. In this event a record is kept of the number of events skipped and ORDER can report the percentage of total events ic has analyzed.

\section{HYDRA}

HYDRA is the largest and most complex task in the MINUS3 package. Its primary function is that of a monitor program and Interactive graphics interface for the user. It therefore utilizes the modifiled Tektronix 4014 terminal and switch interface described in the section on Computer Configuration and shown in figure 1. HYDRA may be used to dynamically monitor information from different sources. These include l-dimensional histogram spectra created by ORDER; live spectra acquired by either the TI analyzers or external memory (see section on Data Acquisition Hardware) or stored spectra from tape. These links are indicated in figure 2.

Information from any of these sources may be monitored at any time without interference. To accomplish this HYDRA utilizes two basic data buffers. The primary buffer is shared with ORDER and normally contains analyzed event data. The secondary buffer is utilized to store additional monitor information as requested. The practical result of this is to permit the user to freely monitor and interact with either the event recorded Information or the live histogram data, or to replay previous results with no effect on acquisition results. Further HYDRA has the ability to store on tape the results it is currently monitoring. Storage and retrieval of information frow tepe way be carried out any time and 
acquiaition need not be halted. To prevent any possible conflicts vith an acquisition tape, HYDRA utilizes its orm tape handling routines similar to those degcribed under RESTOR, to safeguard operation.

Currently HYDRA can simultaneously handle up to 24 l-dimensional spectra frow any data source (i.e., 24 event-recorded projections or 24 spectra from tape), and each spectrum may have different lengths (up to a maximum of 4096 channels). A user may choose to display any one spectrum, all spectra simultaneously, or any selected subset in any order desired. Each spectrum may, independently, be displayed as lipear or $10 \mathrm{~g}$, with the $x$-axis expanded or full scale and with the $y$-axis either automatically nomalized or set manually. These features are, for the most part, controlled and selected dynamically by means of the interactive switches. In addition to these standard analyrer type functlons, HYDRA also offers the following general features.

a) Interactive Backgrounds- Independent background curves may be determined and stored for each spectrum. Each background can be subtracted from its spectrum whenever desired.

b) Peak Fitting--Dynamically selected regions of a spectrum may be fitted with single or multiple Gaissian or asymetric shapes. The results of the fitting may be removed from the data if desired.

c) Gates--The task perwits gating or correlation confitions to be defined or altered dynamically when monitoring event-recorded data.

d) Smoothing or Channel Sums--Histogram data may be displayed a: " "smoothed" spectrum (using a number of possitie swoothing options) or as a 2 or 3 channel sumed spectrum.

e) Spectrum Shifting and Normalization--Any spectrum may be shifted or multiplied by a constant value.

f) Spectrum Addition and Subtraction--Spectra of the same size may be adided or subtracted.

b) Plots and Lists--Any displayed spectrum may be plotted or listed, channel-by-channel. Hard coptes of the screen can also be made by the hardware.

These functions are generally independent and may be combined at will. Thus, for example, a background may be removed from spectrum 1 , spectrum 2 shifted by 10 channels, added to the display of :pectrum 1 minus background, the resulting information smoothed and a plot made.

HYDRA uses a virtual display concept to produce the types of transformations previausly discussed. With t:is techn1que only a single memory buffer is ever actually displayed. All yirtual displays are dynamically created in this buffer as needed. In this manner the basic data are never actually altared. Another graphics technique employed by this task is the simultaneous utilization of both stored and refreshed images. This has the effect of creating two very different intensity levels, and makes the visual comparison of screen information (e.g., the sum of spectra compared with the origina1) much easier to understand.

As a general utility feature, HYDRA may also be requested to run any task existing in the system. The detalls of this option are contained in the discussion of the OVERSEE task in the following section.

\section{SPACE, TIME and OVERSEE}

The four tasks reviewed previous 1 y are all memory resident. The three tasks discussed in this aection are auxillary tasks and are only executed when required. They run in place of HYDRA both in terms of CPU time and, if necessary, memory.

Just as HYDRA handles 1-dimensional spectra, SPACE handles 2-dimensional ones. It is invoked by simply setting a switch on the Tektronix keyboard. SPACE is similar to HYDRA in its theory of operation and utilizes the graphics display and interactive switches for communication. At present it can handle up to nine 2-D spectra and can display one or nore at a time. It permits the removal of background (a constant value); allows the integration of rectangular regions; supports the dynamic setting of gating conditions; and can produce line-printer maps of $2-D$ spectra.

A number of improvements to this task are currently under development. Of particular Importance is the ablitity to set and utilize free-form regions. Such regions could then be used for gating conditlons, integration, and the projection of 1-D Information. Other features under development include log-linear displays; biased 2-D capability; the ability to store and replay 2-D spectra from tape (In a manner analogous to FYDRA); spectrumsmoothing capability; and contour mapping. other possible additions being considered for the future include offering vartous types of 2-D display (e.g., isometric and perspective) with rotational capability, spectra manipulation (addition, subtraction, etc.), non-normal 1-D projections (i.e., not on the $x$ and $y$ axes), and tore complex background manipulation.

The task TIME, shown in figure 2, is currently belng designed. Its purpose is to utilize the graphics terminal to display and manipulate the perlodic or time-dependent information acquired by the computer, either in a real time or a playback mode. Unlike HYDRA and SPACE, no spectra or results actually pre-exist for this task to display. As indicated in the section on RESTOR, perlodic data are witten on disc as well as rape. TIME will interact with the user to determine what Information is of interest. The disc file will then be searched to obtain the necessary raw data. This informarion will then be processed in an appropriate manner to produce the requested displays, One possible request $m 1$ ght be to see the variation of counting rates for a set of scalers at a fixed time interval over a particular 2 hour period. Other requests might be to view the time correlation of a particular current reading or perhaps the variation of a bias voltage with temperature. TIME therefore serves to provide visual display of auxiliary information which may have 
importance to ihe historical or current environment in which the event data vere acquired. Ultimately it is desirable to be able to accept or refect records of event data ascording to conditions In the perlodic data.

As mentloned in the previous section, HYDRA will accept a request to execute any task in the system. As a result of this request HYDRA will load the task OVERJEE. OVERSEE w111 attempt to locate and run the requested task. If it is successful the following conditions will apply: the new task will be given at least $131 \mathrm{~K}$ bytes of memory in which to execute; It will be given the grsphics terminal to use, if desired; and it w1ll be provided all the CPU time not necessary for data acquisition and data sorting. As a precactionary measure OVERSEE will perfodically moititor the user task. If for any reason thi task execution ceases, OVERSEE w1ll resume HYDRA. As a fallsafe measure OVERSEE also monttors one of the interactive switches. This switch must be set while the user task is execuring. If the switch 18 reset, OVERSEE will abort the user task, regardless of what it was doing, remove it from memory and resume HYDRA. In any case BYDRA w111 resume at the polnit 1t was interrupted, but with current, updated spectra. One speclal task a user can request is a standard background. In this case the user may write, edit and store code and then compile, assemble, link, and run 1t. This can be carried su: curing acquisition and all the safeguards discussed above still apply.

\section{Sumary}

A set of tasks has been created to handle the gencral problem of data acquisition at two LBL accelerators. This framework of tasks, now called MINUS3, has been in operation since mid-1976. The on-1ine results to date have been quite satisfactory and the hardware and software standardization have greatly improved and simplified the problem of acquisition. A mafor design criterion for this package was that as much of the traditional off-line analysis capability as posstble be avallable to the on-line user. Although MINUS 3 was designed for on-line data acquisition, and despite the fact that many planned analysis capabilities have not as yet been completed, the package currently serves as the sole off-line analysis program for a considerable number of experimental groups.

$$
\text { It 15, of course, not possible for a single }
$$

package to contain all the analysis capabtlities required by all experiments in an optimum fashion. MINUS3 attempts to satisfy those more-or-less standard requirements which are common to a large number of experiments. For more spectalized requirements it is expected that users will supply their own code. To facilitate this, the concept of parasitic programing has been utilized. Bastcally this means that spectal programs and subroutines are completely witten and maintained by the user. These codes may then be dynamically attached to (or run in conjunction with) the standard package as desired by the user. These user codes then function as independent parasites of the main package. In establishing such a structure it is important that a number of well defined interfaces be established between the main code and potential user codes. The advantage of this approach is that both the user and the main cor may be developed and maintalped Independently of each other. A capability added to the MINUS 3 package can, for example, be immedlately used in conjunction with pre-existing user code. Inter-code interfaces can range from simple disc buffers, written by one code and read by another, to overlapping common regions mapped into user tasks. The optional user cransformation subroutines, described in the section on ORUER, and the ability for users to safely execute and code (see discussion on OVERSEE) are examples of this technique. It therefore appears that the MINUS3 package in conjunction with user-suFplied parasitic code will be atle to provide the benef1ts of standardization, versatility and powerful snalysis capabllity, both on- and of $f-l i n e$, required at both accelerators.

\section{References}

1. i. B. Robinson, F. Gin, F. S. Goulding, Nuclear Instruments and Methods, 62, 237 (1968)

2. L. B. Roblnson, F. Gin, H. Cingoiani, Nuclear Instruments and Methods, 75, 121 (1969)

3. R. V. Poore, D. E. McMillan, R. O. Neison, J. W. Sunfer, IEEE Transactions in Nuclear Sclence, NS-26, No. 1, (Feb. 1979) 